\title{
腺苷酸琥珀酸合成酶双基抑制剂类似物的合成及生物活性
}

\author{
刘斌 孔令青董宏波王明安* \\ (中国农业大学应用化学系 北京 100193)
}

\begin{abstract}
摘要 以丁内酯为原料, 通过缩合、水解、亚胺化、关环、还原、偶联、甲酰化以及脱保护等反应设计并探索了腺苷 酸琥珀酸合成酶双基抑制剂类似物的合成方法, 目标化合物的结构经 ${ }^{1} \mathrm{H}$ NMR 和 HR-ESI-MS 数据进行表征. 初步生物 活性测试结果表明, 目标化合物在 $100 \mu \mathrm{g} / \mathrm{mL}$ 浓度下对油菜和稗草具有较弱的抑制活性.
\end{abstract}

关键词＼cjkstart腺苷酸琥珀酸合成酶; 双基抑制剂; Hadacidin; 合成; 除草活性

\section{Synthesis and Herbicidal Activity of Adenylosuccinate Aynthetase Bisubstrate Hybrid Inhibitor Analogues}

\author{
Liu, Bin Kong, Lingqing Dong, Hongbo Wang, Ming'an* \\ (Department of Applied Chemistry, China Agricultural University, Beijing 100193)
}

\begin{abstract}
The adenylosuccinate synthetase (AdSS) bisubstrate hybrid inhibitor analogues 5-(4-hydroxybenzyl)- $N^{3}$ [5-ethoxycarbonyl-4-( $N$-formylhydroxyamino)pentyl]-hydantoin benzoates containing the structural moiety of hadacidin were designed and synthesized via Claisen condensation, hydrolysis decarbosylation, imidization, acid-catalyzed ring closure, borane reduction, Mitsunobu coupling reaction, formylation and deprotection used 1,4-butyrolactone as raw materials. Their structures were characterized with ${ }^{1} \mathrm{H}$ NMR and HR-ESI-MS data. The preliminary and greenhouse test results showed that these compounds only exhibited weak inhibition against E. rusgalli and B. campestris at the concentration of $100 \mu \mathrm{g} / \mathrm{mL}$.
\end{abstract}

Keywords adenylosuccinate synthetase; bisubstrate hybrid inhibitor; hadacidin; synthesis; herbicidal activities

腺苷酸琥珀酸合成酶(Adenylosuccinate synthetase, AdSS) 是存在于植物、细菌以及肿瘤细胞中嘌呤生物合 成前体的一种关键酶 ${ }^{[1 \sim 4]}$, 在药物化学和农药化学研究 中具有重要的作用. 近年来发现一些 AdSS 酶抑制剂已 经用作抗肿瘤药物的有效成分, 而针对 AdSS 酶的抑制 剂类抗肿瘤药物开发是国际上的一个研究热点 ${ }^{[2,5]}$, 在 此本文不必细述. 早期发现 AdSS 酶抑制剂 hadacidin 可 以显著抑制植物的生长发育 ${ }^{[6]}$, 但是一直未得到足够关 注，自从 Nakajima 等 ${ }^{[7]}$ 发现天然产物 hydantocidin 具有 高效的非选择性除草活性以来，人们探索了 hydantocidin 的全合成方法、作用机制、作用靶标及其 与靶标 AdSS 酶复合物的晶体结构, 推动了 AdSS 酶抑 制剂在新农药创制中的研究步伐, 本文作者对此已作过 综述 ${ }^{[8]}$. 本课题组 ${ }^{[9]}$ 曾利用距离几何学、分子间氢键等分 析肌苷单磷酸(IMP)识别 AdSS 的活性位点, 同时利用分
子对接、分子力学优化及分子动力学模拟等方法进行理 论预测, 结果显示 Hydantocidin 5'-phosphate 与底物 IMP 竞争而产生抑制活性，与 Walters 等 ${ }^{[10]}$ 报道的抑制机制 一致，同时指出磷酸基团在抑制剂与酶蛋白的结合中有 重要的作用，在此基础上我们设计合成了一些新的 AdSS 酶抑制剂, 生物活性测试结果表明部分化合物显 示出良好的除草活性 ${ }^{[11]}$.

双基或多基抑制剂(bisubstrate hybrid inhibitor，or multisubstrate inhibitor)的概念最早在药物化学领域中由 Lienhard 等 ${ }^{[2]}$ 提出. 近年来随着越来越多蛋白分子的三 维溶液结构和晶体结构得到阐明, 为相关的生理作用机 理提供了分子水平上的依据, 极大地推动了药物分子的 研究和发现步伐. 从酶蛋白结构出发并结合计算机技 术，不断有新的酶抑制剂的设计思路出现，其中双基抑 制剂(bisubstrate hybrid inhibitor)的设计策略在不少药物

*E-mail: wangma@cau.edu.cn

Received June 24, 2015; revised August 17, 2015; published online August 26, 2015.

Project supported by National Natural Science foundation of China (No. 20772150) and National KeyTechnologies R\&D Program of China (No. 2011BAE06B04).

国家自然科学基金(No. 20772150)和国家十二五科技支撑计划(No. 2011BAE06B04)资助项目. 
靶标的研究中得到推广使用 ${ }^{[13]}$. 在 AdSS 酶抑制剂的研 究中, 先后由 Hanessian ${ }^{[14]}$ 和 Wrasidlo 等 ${ }^{[5,15]}$ 合成了 AdSS 酶双基抑制剂, 结果发现它们的 AdSS 酶抑制活性 得到显著提高, 如 Hanessian 等 ${ }^{[14]}$ 将 hydantocidin 与 hadacidin 交联在一起合成的 AdSS 酶双基抑制剂，其对 大肠杆菌 AdSS 的抑制活性提高到 hydantocidin 的 15.7 倍, hadacidin 的 81.4 倍, 其对小麦 AdSS 的抑制活性提 高到 hydantocidin 的 6.8 倍, hadacidin 的 60 倍, 但是关 键中间体的合成原料难得、试剂昂贵、分离困难, 至今 未能实现它们的工业化生产. 继续课题组 ${ }^{[11]}$ 已有的研究 工作, 为了发现具有较高除草活性的先导化合物, 本文 将双基抑制剂的理念用于 AdSS 酶抑制剂的分子设计, 将 AdSS 酶抑制剂 hadacidin 的结构片段设计在前期发现 的一些分子中, 并探讨该类化合物新的合成策略, 化合 物的合成路线如 Scheme 1 所示.

\section{1 结果与讨论}

\section{1 目标化合物的设计及合成方法}

为了高效的合成 AdSS 双基抑制剂类似物，参照文 献我们最初设计合成化合物 7 作为关键中间体(Scheme 2)，但是在顺利制备得到化合物 4 和 5-溴-2-羊基亚胺基 戊酸的顺反异构体混合物 $\mathbf{5}$ 后，由于混合物 $\mathbf{5}$ 难以溶解, 很难进行下一步的氧基硼氢化钠还原反应只好放弃. 于 是又设计合成化合物 9 作为关键中间体再转化成中间体 7, 这条路线也是在顺利制备得到化合物 8 和 5-羟基-2差基亚胺基戊酸的顺反异构体混合物 9 后，同样难以顺 利地进行氰基硼氢化钠还原反应也只好放弃. 考虑到化 合物的极性较大对分离造成的困难，可以将羟基用苄基 保护起来有利于分离纯化, 于是又设计将化合物 8 与茮 氧羟胺反应，结果证明得到了化合物 $\mathbf{2}$ 和 11 的混合物,
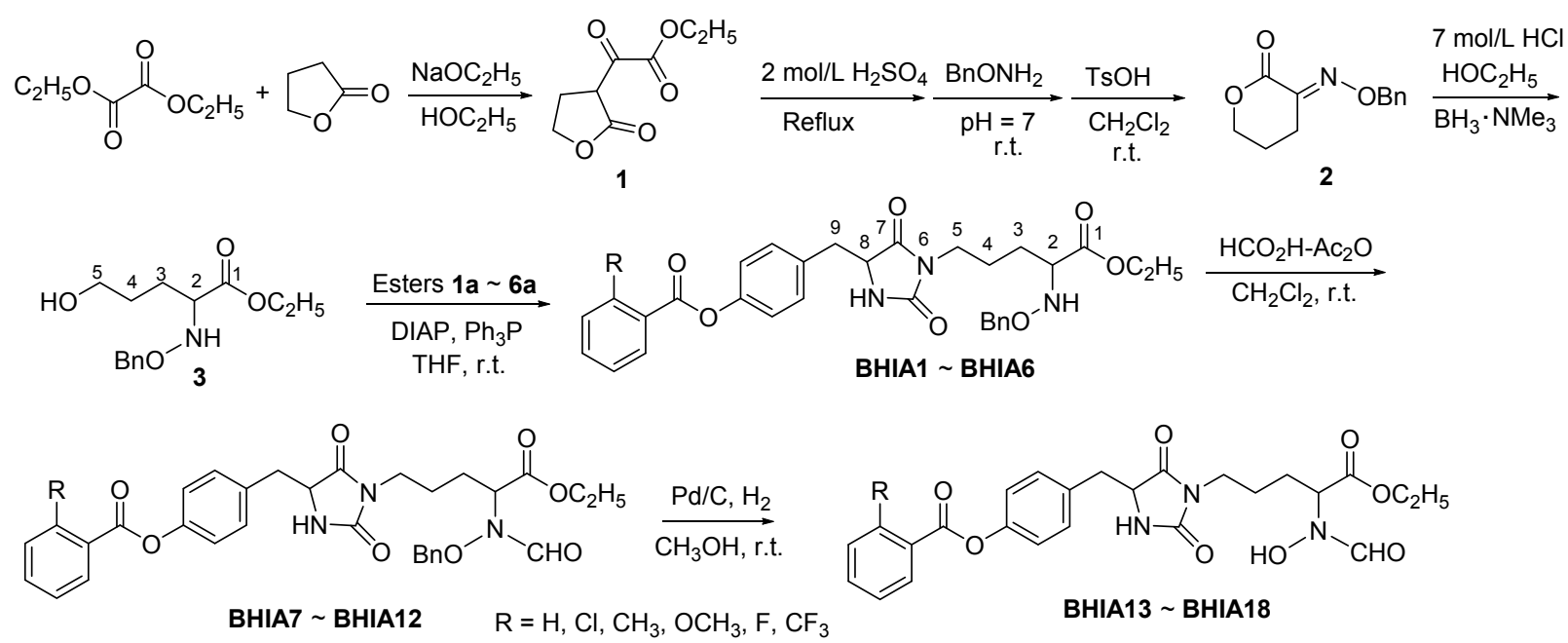

图式 1 AdSS 双基抑制剂类似物的合成路线

Scheme 1 Synthetic routes of AdSS bisubstrate hybrid inhibitor analogues
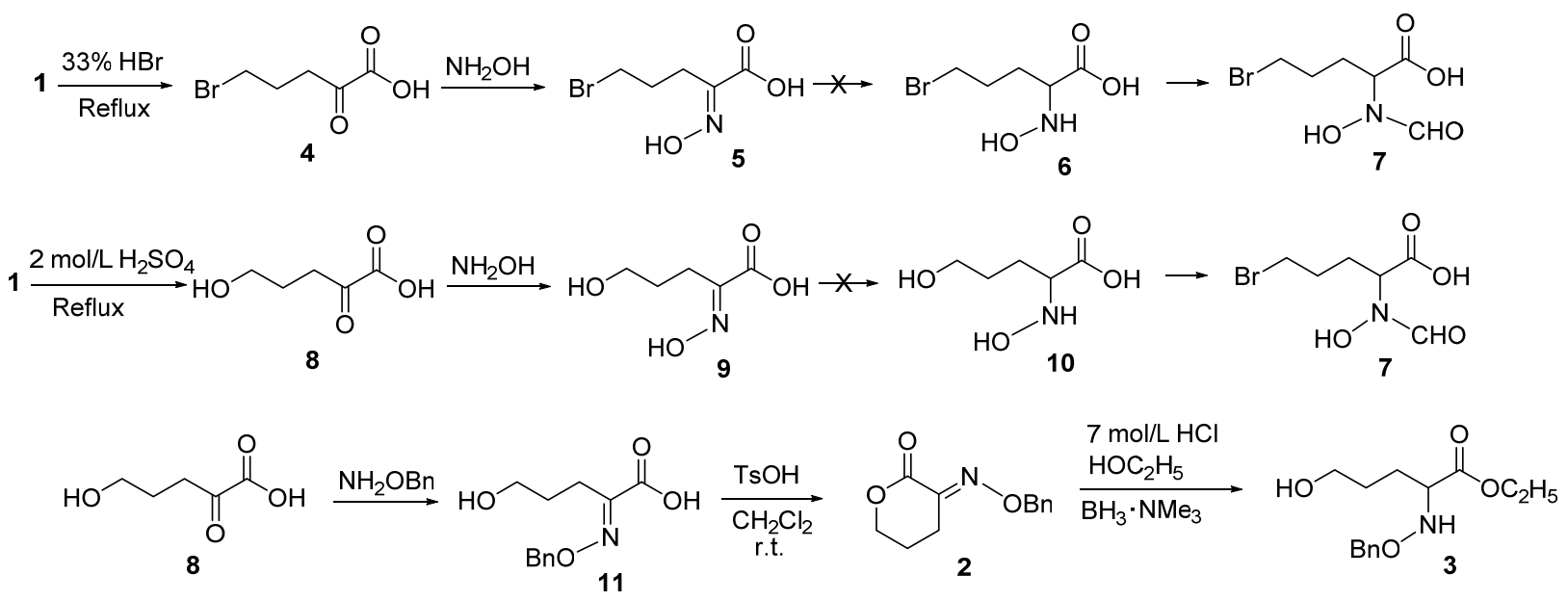

图式 2 关键中间体的合成

Scheme 2 Synthesis of key intermediates 
后来通过加入苯甲醇并 DCC/DMAP 体系进行茮酯化以 进一步降低极性, 结果意外地发现全部转化成了化合物 2 , 在 $\mathrm{pH}$ 为 4 左右的条件下化合物 $\mathbf{2}$ 进行氰基硼氢化钠 还原反应时又得到化合物 $\mathbf{2}$ 和 $\mathbf{1 1}$ 的甲酯的混合物, 双键 没有变化.

从前面的亚胺化反应、芐酯化反应和还原反应的处 理过程看, 2-芐氧基亚胺基-1,5-戊内酯(2)较 5-着基-2-芐 氧基亚胺基戊酸(11)及其酯更加稳定, 但是在酸性条件 下 2 容易开环为 11, 而在后处理过程中的加热可能导致 11 重新关环为内酯 2 , 说明化合物 2 是一个稳定的中间 体. 在此基础上, 作者尝试将化合物 $\mathbf{2}$ 和 $\mathbf{1 1}$ 的混合物分 别用对甲苯磺酸吡啶盐、浓盐酸以及对甲苯磺酸在室温 和加热回流条件下催化关环, 结果发现对甲苯磺酸在室 温就可以全部将它们转化为内酯 $\mathbf{2}$, 而对甲苯磺酸吡啶 盐和浓盐酸则难以实现完全转化. 据此设计了从化合物 1 到化合物 2 的三步一锅法反应, 结果证明该方法非常 成功, 三步反应的总收率达到了 $70 \%$. 如上所述化合物 2 不能被氧基嗍氢化钠还原, 经文献查阅发现嗍烷吡啶 络合物 ${ }^{[16]}$ 和嗍烷三甲胺络合物 ${ }^{[17]}$ 两种还原剂能够将羧 酸酯的 $\alpha$-芐氧亚氨基还原为芐氧氨基，而化合物 2 在酸 性甲醇体系中也会开环生成 11 的甲酯, 故采用酸性条 件下以硼烷三甲胺络合物为还原剂在乙醇体系中进行 酯交换和还原反应，结果顺利地制得了关键化合物 3 . 化合物 3 与原有 ${ }^{[11]}$ 的中间体酯 $1 \mathrm{a} \sim 6 \mathbf{a}$ 进行偶联反应获 得了腺苷酸琥珀酸合成酶双基抑制剂类似物 BHIA1

BHIA6, 然后再 BHIA1 BHIA6 经甲酸-乙酸䣶甲酰化 反应得到双基抑制剂类似物 BHIA7 BHIA12, 后者经 $\mathrm{Pd} / \mathrm{C}$ 氢化脱除苄基最后得到了目标化合物 BHIA13 BHIA18. 我们曾经通过使用不同的碱试图水解 BHIA13 BHIA18 得到末端为羧酸的化合物, 结果末 端酯基和母体酚酯部分均水解脱掉了, 这些化合物 都没有生物活性, 所以保留母体酚酯部分是必要的. 鉴于化合物 $\mathbf{3}$ 的手性碳原子构型可能对目标化合物生物 活性产生较大影响 ${ }^{[14]}$, 在本文合成了消旋体的基础上, 作者对化合物 $\mathbf{2}$ 的不对称还原反应进行了有益探索, 相 关结果将另文发表.

\section{2 目标化合物的图谱分析}

关键中间体化合物 3 的 ${ }^{1} \mathrm{H} \mathrm{NMR}$ 谱中: 由于 2 位碳 为手性原子, 3,4 位碳上质子不等价在 $\delta 1.78 \sim 1.47$ 范围 内为复杂的多重峰, 2 位碳和 5 位碳上的三个质子在 $\delta$ $3.63 \sim 3.53$ 范围内也为复杂的多重峰, 其余苯环、亚甲 基及乙酯质子未见异常; 中间体化合物 $\mathbf{3}$ 与酯 $\mathbf{1 a} \sim 6 \mathbf{6}$ 偶联获得的双基抑制剂类似物 BHIA1 BHIA6, 除苯 环、亚甲基及乙酯质子易于识别外, 通过与中间体比较 发现 2, 3, 4 位碳上质子没有明显位移, 5 位碳上的二个
质子则向高场移动以三重峰形式出现在 $\delta 3.45,8$ 位碳上 的质子以双二重峰形式出现在 $\delta 4.25$ 并与乙酯的亚甲基 质子峰重叠在一起, 9 位碳上的两个质子磁不等价分别 在 $\delta 3.26$ 和 2.88 以双二重峰形式出现; 当化合物 BHIA1 BHIA6 甲酰化后得到的 BHIA7 $\sim$ BHIA12, 其 ${ }^{1} \mathrm{H}$ NMR 谱与 BHIA1 BHIA6 十分相似，但是会导致 2 位和苠基的亚甲基质子位移向低场移动，同时由于甲酰 胺的部分双键性质使得谱图更加复杂，这与文献报道的 类似甲酰胺衍生物复杂的 ${ }^{1} \mathrm{H}$ NMR 谱相似 ${ }^{[18]}$; 当化合物 BHIA7 BHIA12 脱除苄基保护后得到的 BHIA13 BHIA18, 其 ${ }^{1} \mathrm{H}$ NMR 谱与 BHIA7 $\sim$ BHIA12 相比较十 分相似，只是减少了芳基的质子峰. 所有化合物的 HR-ESI-MS 谱中, 均可以获得它们的准分子离子 $[\mathrm{M}+$ $\mathrm{H}]^{+}$峰的精确质量并与理论计算值吻合, 如化合物 BHIA13 的 $\mathrm{HR} \mathrm{MS} m / z \mathrm{C}_{25} \mathrm{H}_{27} \mathrm{~N}_{3} \mathrm{O}_{8}[\mathrm{M}+\mathrm{H}]^{+}$, 计算值为 498.1871, 测定值为 498.1863 .

\section{3 目标化合物的除草活性}

选择以油菜(Brassica campestris) 和稗草 (Echinochloa crusgalli) 作为靶标植物, 参照前文 ${ }^{[11]}$ 采用油菜平 皿法和稗草小杯法测试了化合物的除草活性, 并选择以 油菜、稗草、苋菜(Amaranthus tricolor)和马塘(Digitaria sanguinalis) 为靶标植物对化合物以土壤处理和茎叶处 理方式进行了盆栽实验，以草甘膦为阳性对照，表 1 显 示了化合物 $1 \mathrm{a} \sim 6 \mathrm{a}$ 和目标化合物 BHIA13 $\sim$ BHIA18 对 它们的除草活性，由于中间化合物 BHIA1 BHIA6 和 BHIA7 $\sim$ BHIA12 分子中缺少活性基团甲酰基或含有茮 基保护基, 并未测试它们的除草活性. 从表 1 可以看出, 在平皿法和小杯法测试结果中，化合物 $1 \mathrm{a} \sim 6 \mathrm{a}$ 和 BHIA13 BHIA18 对油菜显示了一定的抑制活性但抑 制率都不高，而它们对稗草基本没有抑制活性; 而在盆 栽实验结果中, 化合物 $1 \mathrm{a} \sim 6 \mathrm{a}$ 对油菜、苋菜和马塘完全 无抑制活性, BHIA13 BHIA18 对油菜、苋菜和马塘显 示出较弱的抑制活性，化合物 $1 \mathrm{a} \sim 6 \mathbf{6}$ 和 BHIA13 BHIA18 对稗草均显示了一定的抑制活性. 我们可以看 出, 化合物 1a $\sim 6 \mathrm{a}$ 与 BHIA13 $\sim$ BHIA18 相比, 它们的 除草活性没有明显改善, 这可能与 BHIA13 BHIA18 的 2 位碳为手性原子但本文合成的是消旋体混合物有 关，也与分子中 hadacidin 片段的羒基为酯而不是羧基 以及与母体化合物 $1 \mathrm{a} \sim 6 \mathbf{6}$ 的抑制活性较差密切相关. 这些结果提示，在 AdSS 双基抑制剂类化合物的分子设 计中, 一方面将 hadacidin 片段设计为光活性的结构将 有利于生物活性的提高 ${ }^{[14,15]}$, 另一方面母体化合物本身 生物活性的好坏将决定设计合成的双基抑制剂类化合 物生物活性的优劣. 
表 1 化合物 BHIA13 18 的除草活性(抑制率/\%)

Table 1 The herbicidal activities of target compounds BHIA13 $\sim 18$ (inhibition rate/\%)

\begin{tabular}{|c|c|c|c|c|c|c|c|c|}
\hline \multirow{2}{*}{ Compd. } & \multicolumn{2}{|c|}{ 油菜 } & \multicolumn{2}{|c|}{ 稗草 } & \multirow{2}{*}{ 油菜 } & \multirow{2}{*}{ 苋菜 } & \multirow{2}{*}{ 稗草 } & \multirow{2}{*}{ 马唐 } \\
\hline & $10 \mu \mathrm{g} / \mathrm{mL}$ & $100 \mu \mathrm{g} / \mathrm{mL}$ & $10 \mu \mathrm{g} / \mathrm{mL}$ & $100 \mu \mathrm{g} / \mathrm{mL}$ & & & & \\
\hline Ester 1a & 0 & 5.9 & 0 & 0 & 0 & 0 & 0 & 0 \\
\hline Ester $\mathbf{2 a}$ & 0 & 0 & 0 & 0 & 0 & 0 & 5.0 & 0 \\
\hline Ester 3a & 13.5 & 25.5 & 0 & 0 & 0 & 0 & 10.0 & 0 \\
\hline Ester 4a & 13.2 & 32.3 & 0 & 5.0 & 0 & 0 & 12.0 & 0 \\
\hline Ester $\mathbf{5 a}$ & 0 & 1.0 & 0 & 3.0 & 0 & 0 & 10.0 & 0 \\
\hline Ester 5a & 11.8 & 33.8 & 0 & 0 & 0 & 0 & 3.0 & 0 \\
\hline BHIA13 & 0 & 10.4 & 0 & 2.0 & 5.0 & 0 & 15.0 & 5.0 \\
\hline BHIA14 & 0 & 17.4 & 0 & 0 & 5.0 & 0 & 0 & 0 \\
\hline BHIA15 & 0 & 10.1 & 0 & 3.0 & 5.0 & 10.0 & 0 & 0 \\
\hline BHIA16 & 0 & 9.0 & 0 & 3.0 & 0 & 5.0 & 20.0 & 0 \\
\hline BHIA17 & 0 & 4.5 & 0 & 0 & 0 & 0 & 0 & 0 \\
\hline BHIA18 & 0 & 8.7 & 0 & 0 & 10.0 & 10.0 & 10.0 & 0 \\
\hline
\end{tabular}

\section{2 实验部分}

\section{1 仪器与试剂}

Yanagimoto 显微熔点仪(温度计未校正); Bruker DPX $300 \mathrm{MHz}$ 核磁共振仪, TMS 为内标, $\mathrm{CDCl}_{3}$ 为溶剂, 化学位移用 $\delta$ 表示; Agilent 1100 LC-MSD-Trap 质谱仪 (ESI-MS), Brüker Apex II 高分辨质谱仪. 本实验所用柱 层析硅胶为青岛海洋化工有限公司生产(200～300 目), 所用试剂均为国产或进口分析纯, 无水溶剂用常规方法 干燥处理.

\section{2 化合物的合成}

\subsubsection{2-乙氧草酰基-1,4-丁内酯(1)的合成}

中间体 1 参照文献[19]方法合成, 无色油状液体, 收率为 $84 \% .{ }^{1} \mathrm{H} \mathrm{NMR}\left(300 \mathrm{MHz}, \mathrm{CDCl}_{3}\right) \delta: 10.92(\mathrm{~s}, 1 \mathrm{H})$, 4.50 (t, $J=7.5 \mathrm{~Hz}, 2 \mathrm{H}), 4.27$ (q, $J=7.2 \mathrm{~Hz}, 2 \mathrm{H}), 3.30$ (t, $J=7.5 \mathrm{~Hz}, 2 \mathrm{H}), 1.41(\mathrm{t}, J=7.2 \mathrm{~Hz}, 3 \mathrm{H})$.

\subsubsection{2-芐氧亚氨基-1,5-戊内酯(2)的合成}

在 $500 \mathrm{~mL}$ 的三口瓶中加入 $12.1 \mathrm{~g}(65 \mathrm{mmol}) 2$-乙氧 草酰基-1,4-丁内酯, 再加入 $80 \mathrm{~mL} 2 \mathrm{~mol} / \mathrm{L} \mathrm{H}_{2} \mathrm{SO}_{4}$ 溶液 中, 摚拌下加热回流 $5 \mathrm{~h}$. 冷却后, 用 $20 \% \mathrm{NaOH}$ 溶液调 $\mathrm{pH}$ 至 7 8, 缓慢滴加 $60 \mathrm{mmol}$ 芐基羟胺溶液 (4 h), 室温 搅拌过夜, TLC 监测芐基羟胺原料点消失, 调节 $\mathrm{pH}$ 至 12 , 乙醚洗涤两次后, 再将水相调至 $\mathrm{pH}=1$, 用 $1.2 \mathrm{~L}$ 二 氯甲烷萃取三次, 有机相经无水硫酸钠干燥, 过滤除去 固体, 有机相直接加入 $2000 \mathrm{~mL}$ 单口瓶中, 再加入 200 $\mathrm{mg}$ 对甲苯磺酸, 室温搅拌过夜, 减压浓缩至 $500 \mathrm{~mL}$, 用饱和碳酸氢钠溶液和饱和氯化钠溶液各洗一次, 有机 相经无水硫酸钠干燥, 过滤除去固体, 母液经旋蒸浓缩 得微黄色油状产物 $29.95 \mathrm{~g}$, 收率 70\%. ${ }^{1} \mathrm{H}$ NMR (300 $\left.\mathrm{MHz}, \mathrm{CDCl}_{3}\right) \delta: 7.39 \sim 7.34(\mathrm{~m}, 5 \mathrm{H}), 5.37(\mathrm{~s}, 2 \mathrm{H}), 4.35$ (t, $J=5.2 \mathrm{~Hz}, 2 \mathrm{H}), 2.77(\mathrm{t}, J=6.9 \mathrm{~Hz}, 2 \mathrm{H}), 2.03 \sim 1.95(\mathrm{~m}$,
2H). HR-MS calcd for $\mathrm{C}_{12} \mathrm{H}_{14} \mathrm{NO}_{3}[\mathrm{M}+\mathrm{H}]^{+}$220.0968, found 220.0968 .

\subsubsection{2-芳氧氨基-5-羟基戊酸乙酯(3)的合成}

在 $150 \mathrm{~mL}$ 的圆底烧瓶中将 $2.19 \mathrm{~g}(10 \mathrm{mmol}) 2$-芐氧 亚氨基-1,5-戊内酯溶于 $40 \mathrm{~mL}$ 无水乙醇, 加入 $25 \mathrm{~mL} 7$ $\mathrm{mol} / \mathrm{L} \mathrm{HCl} / \mathrm{EtOH}$ 溶液, 常温搅拌 $30 \mathrm{~min}$, 加入 $1.15 \mathrm{~g} \mathrm{(15}$ $\mathrm{mmol}$ )三甲胺硼烷络合物( $(95 \%)$, 常温搅拌 $8 \mathrm{~h}$ 后, 再补 加 $1.15 \mathrm{~g}$ 三甲胺硼烷络合物继续摚拌反应, TLC 监测至 2-芐氧亚氨基-1,5-戊内酯消失, 停止搅拌, 在冰水浴中 以 $20 \%$ 氢氧化钠溶液调 $\mathrm{pH}$ 至 8 , 用 $200 \mathrm{~mL}$ 二氯甲烷萃 取三次, 有机相经无水硫酸钠干燥, 过滤除去固体, 母 液浓缩后经硅胶柱层析分离, 以二氯甲烷/乙醇体系为 洗脱剂进行梯度洗脱得产物无色油状物 $31.56 \mathrm{~g}$, 收率 $58 \%$. ${ }^{1} \mathrm{H}$ NMR $\left(300 \mathrm{MHz}, \mathrm{CDCl}_{3}\right) \delta: 7.36 \sim 7.30(\mathrm{~m}, 5 \mathrm{H})$, $4.71(\mathrm{~s}, 2 \mathrm{H}), 4.22(\mathrm{q}, J=7.1 \mathrm{~Hz}, 2 \mathrm{H}), 3.63 \sim .53(\mathrm{~m}, 3 \mathrm{H})$, $1.78 \sim 1.47(\mathrm{~m}, 4 \mathrm{H}), 1.29$ (t, $J=7.1 \mathrm{~Hz}, 3 \mathrm{H}) ;{ }^{13} \mathrm{C}$ NMR $(75$ $\left.\mathrm{MHz}, \mathrm{CDCl}_{3}\right) \delta: 173.8,137.5,128.4,128.3,127.8,76.0$, 63.6, 62.2, 61.0, 29.6, 26.7, 14.2. HR-MS calcd for $\mathrm{C}_{14} \mathrm{H}_{22} \mathrm{NO}_{4}[\mathrm{M}+\mathrm{H}]^{+} \quad 268.1543$, found 268.1542 .

2.2.4 2-芐氧氨基-5-羟基戊酸乙酯(3)的 Mitsunobu 偶 联反应

将 $0.59 \mathrm{~g}(2.2 \mathrm{mmol}) 2$-芐氧氨基-5-差基戊酸乙酯、 $0.66 \mathrm{~g}$ (2 mmol) 5-(4-差基苄基)-2,4-咪唑啉二酮邻氟苯 甲酸酯及 $0.74 \mathrm{~g}(2.8 \mathrm{mmol})$ 三苯基膦 $\left(\mathrm{PPh}_{3}\right)$ 依次加入到 $100 \mathrm{~mL}$ Schlenk 瓶中, 在氮气保护下加入重蒸四氢呋喃 $40 \mathrm{~mL}$, 常温下搅拌溶解. 用注射器分次加入 $0.58 \mathrm{~g}$ (2.8 $\mathrm{mmol}$ )偶氮二甲酸二异丙酯(DIAD), 开始加入 DIAD 时 体系变成黄色, 搅拌后黄色裉去, 再继续加入 DIAD, 重复直至 DIAD 加完(约 $20 \mathrm{~min}$ ), 体系呈黄色, 常温摚 拌 $12 \mathrm{~h}, \mathrm{TLC}$ 监测直至 5-(4-羊基芳基)-2,4-咪唑啉二酮邻 氟苯甲酸酯荧光点消失, 停止搅拌反应, 减压脱除溶剂, 
残余物经硅胶柱层析分离(200 300 目), 以石油梄/无 水乙醇为洗脱剂进行梯度洗脱, 浓缩得 $0.68 \mathrm{~g}$ 无色粘稠 液体 BHIA3, 收率 $59 \%$. 采用相同方法合成化合物 BHIA1 BHIA6.

5-对羟基苄基- $N^{3}$-(5-乙氧羰基-4-芐氧氨基)戊基2,4-咪唑二酮苯甲酸酯(BHIA1): 无色油状液体, 收率 $61 \% .{ }^{1} \mathrm{H}$ NMR $\left(300 \mathrm{MHz}, \mathrm{CDCl}_{3}\right) \delta: 8.19 \sim 8.16(\mathrm{~m}, 2 \mathrm{H})$, $7.66 \sim 7.61(\mathrm{~m}, 1 \mathrm{H}), 7.52 \sim 7.47(\mathrm{~m}, 2 \mathrm{H}), 7.33 \sim 7.15(\mathrm{~m}$, 9H), 5.95 (brs, $1 \mathrm{H}), 5.68$ (d, $J=2.8 \mathrm{~Hz}, 1 \mathrm{H}), 4.67$ (s, 2H), $4.23 \sim 4.16(\mathrm{~m}, 3 \mathrm{H}), 3.57(\mathrm{t}, J=6.5 \mathrm{~Hz}, 1 \mathrm{H}), 3.43$ (t, $J=$ $6.0 \mathrm{~Hz}, 2 \mathrm{H}), 3.27(\mathrm{dd}, J=14.0,3.7 \mathrm{~Hz}, 1 \mathrm{H}), 2.91 \sim 2.83$ $(\mathrm{m}, 1 \mathrm{H}), 1.65 \sim 1.39(\mathrm{~m}, 4 \mathrm{H}), 1.27(\mathrm{t}, J=7.1 \mathrm{~Hz}, 3 \mathrm{H})$. HR-MS calcd for $\mathrm{C}_{31} \mathrm{H}_{34} \mathrm{~N}_{3} \mathrm{O}_{7}[\mathrm{M}+\mathrm{H}]^{+}$560.2391, found 560.2381 .

5-对羟基芳基- $N^{3}$-(5-乙氧羰基-4-芐氧氨基)戊基2,4-咪唑二酮-2-甲氧基苯甲酸酯(BHIA2): 无色油状液 体, 收率 96\%. ${ }^{1} \mathrm{H}$ NMR (300 MHz, $\left.\mathrm{CDCl}_{3}\right) \delta: 8.02 \sim 7.99$ $(\mathrm{m}, 1 \mathrm{H}), 7.57 \sim 7.51(\mathrm{~m}, 1 \mathrm{H}), 7.32 \sim 7.15(\mathrm{~m}, 9 \mathrm{H}), 7.05 \sim$ $7.00(\mathrm{~m}, 2 \mathrm{H}), 5.95(\mathrm{~d}, J=9.6 \mathrm{~Hz}, 1 \mathrm{H}), 5.75(\mathrm{~d}, J=2.2 \mathrm{~Hz}$, $1 \mathrm{H}), 4.67(\mathrm{~s}, 2 \mathrm{H}), 4.23 \sim 4.16(\mathrm{~m}, 3 \mathrm{H}), 3.92(\mathrm{~s}, 3 \mathrm{H}), 3.57$ (t, $J=7.0 \mathrm{~Hz}, 1 \mathrm{H}), 3.42(\mathrm{t}, J=6.8 \mathrm{~Hz}, 2 \mathrm{H}), 3.25$ (dd, $J=$ $14.0,3.7 \mathrm{~Hz}, 1 \mathrm{H}), 2.90 \sim 2.81(\mathrm{~m}, 1 \mathrm{H}), 1.69 \sim 1.39(\mathrm{~m}$, $4 \mathrm{H}), 1.26(\mathrm{t}, J=7.1 \mathrm{~Hz}, 3 \mathrm{H})$. HR-MS calcd for $\mathrm{C}_{32} \mathrm{H}_{36} \mathrm{~N}_{3} \mathrm{O}_{8}$ $[\mathrm{M}+\mathrm{H}]^{+}$590.2497, found 590.2486.

5 -对羟基苄基- $N^{3}$-(5-乙氧羰基-4-芐氧氨基)戊基2,4-咪唑二酮-2-氟苯甲酸酯(BHIA3): 无色油状液体, 收率 59\%. ${ }^{1} \mathrm{H}$ NMR $\left(300 \mathrm{MHz}, \mathrm{CDCl}_{3}\right) \delta: 8.10 \sim 8.05(\mathrm{~m}$, $1 \mathrm{H}), 7.60 \sim 7.57(\mathrm{~m}, 1 \mathrm{H}), 7.32 \sim 7.16(\mathrm{~m}, 11 \mathrm{H}), 6.05$ (brs, $1 \mathrm{H}), 5.88$ (d, $J=2.9 \mathrm{~Hz}, 1 \mathrm{H}), 4.67(\mathrm{~s}, 2 \mathrm{H}), 4.23 \sim$ $4.16(\mathrm{~m}, 3 \mathrm{H}), 3.57(\mathrm{t}, J=7.0 \mathrm{~Hz}, 1 \mathrm{H}), 3.42(\mathrm{t}, J=6.7 \mathrm{~Hz}$, $2 \mathrm{H}), 3.26(\mathrm{dd}, J=14.0,3.7 \mathrm{~Hz}, 1 \mathrm{H}), 2.95 \sim 2.82(\mathrm{~m}, 1 \mathrm{H})$, $1.58 \sim 1.44(\mathrm{~m}, 4 \mathrm{H}), 1.26(\mathrm{t}, J=7.1 \mathrm{~Hz}, 3 \mathrm{H})$. HR-MS calcd for $\mathrm{C}_{31} \mathrm{H}_{33} \mathrm{FN}_{3} \mathrm{O}_{7}[\mathrm{M}+\mathrm{H}]^{+}$578.2297, found 578.2289.

5 -对羟基苄基- $N^{3}$-(5-乙氧羰基-4-芐氧氨基)戊基2,4-咪唑二酩-2-氯苯甲酸酯(BHIA4): 无色油状液体, 收率 $81 \% .{ }^{1} \mathrm{H}$ NMR $\left(300 \mathrm{MHz}, \mathrm{CDCl}_{3}\right) \delta: 8.04 \sim 8.00(\mathrm{~m}$, $1 \mathrm{H}), 7.53 \sim 7.46(\mathrm{~m}, 2 \mathrm{H}), 7.40 \sim 7.18(\mathrm{~m}, 10 \mathrm{H}), 6.04$ (brs, $1 \mathrm{H}), 5.69$ (brs, $1 \mathrm{H}), 4.67$ (s, 2H), $4.23 \sim 4.15(\mathrm{~m}, 3 \mathrm{H})$, $3.58(\mathrm{t}, J=7.0 \mathrm{~Hz}, 1 \mathrm{H}), 3.43$ (t, $J=6.8 \mathrm{~Hz}, 2 \mathrm{H}), 3.27$ (dd, $J=14.0,3.6 \mathrm{~Hz}, 1 \mathrm{H}), 2.92 \sim 2.84(\mathrm{~m}, 1 \mathrm{H}), 1.64 \sim 1.41(\mathrm{~m}$, 4H), 1.26 (t, $J=7.5 \mathrm{~Hz}, 3 \mathrm{H})$. HR-MS calcd for $\mathrm{C}_{31} \mathrm{H}_{33} \mathrm{ClN}_{3} \mathrm{O}_{7}[\mathrm{M}+\mathrm{H}]^{+}$594.2002, found 594.1992.

5-对羟基苄基- $N^{3}$-(5-乙氧羰基-4-芐氧氨基)戊基2,4-咪唑二酮-2-甲基苯甲酸酯(BHIA5): 无色油状液体, 收率 65\%. ${ }^{1} \mathrm{H}$ NMR $\left(300 \mathrm{MHz}, \mathrm{CDCl}_{3}\right) \delta: 8.16 \sim 8.12(\mathrm{~m}$,
$1 \mathrm{H}), 7.50 \sim 7.44(\mathrm{~m}, 1 \mathrm{H}), 7.32 \sim 7.14(\mathrm{~m}, 11 \mathrm{H}), 6.00$ (brs, $1 \mathrm{H}$ ), 5.76 (brs, $1 \mathrm{H}), 4.67$ (s, $2 \mathrm{H}), 4.23 \sim 4.16(\mathrm{~m}, 3 \mathrm{H})$, $3.57(\mathrm{t}, J=7.0 \mathrm{~Hz}, 1 \mathrm{H}), 3.43(\mathrm{t}, J=6.8 \mathrm{~Hz}, 2 \mathrm{H}), 3.27(\mathrm{dd}$, $J=14.0,3.6 \mathrm{~Hz}, 1 \mathrm{H}), 2.91 \sim 2.83(\mathrm{~m}, 1 \mathrm{H}), 2.65(\mathrm{~s}, 3 \mathrm{H})$, $1.64 \sim 1.38(\mathrm{~m}, 4 \mathrm{H}), 1.27(\mathrm{t}, J=7.1 \mathrm{~Hz}, 3 \mathrm{H})$. HR-MS calcd for $\mathrm{C}_{32} \mathrm{H}_{36} \mathrm{~N}_{3} \mathrm{O}_{7}[\mathrm{M}+\mathrm{H}]^{+}$574.2548, found 574.2539.

5-对羟基苄基- $N^{3}$-(5-乙氧羰基-4-芐氧氨基)戊基2,4-咪唑二酮-2-三氟甲基苯甲酸酯(BHIA6): 无色油状 液体, 收率 57\%. ${ }^{1} \mathrm{H}$ NMR $\left(300 \mathrm{MHz}, \mathrm{CDCl}_{3}\right) \delta:$ 7.98 $7.94(\mathrm{~m}, 1 \mathrm{H}), 7.83 \sim 7.80(\mathrm{~m}, 1 \mathrm{H}), 7.70 \sim 7.65(\mathrm{~m}, 2 \mathrm{H})$, $7.32 \sim 7.19(\mathrm{~m}, 9 \mathrm{H}), 6.02$ (brs, $1 \mathrm{H}), 5.75(\mathrm{~d}, J=3.0 \mathrm{~Hz}$, $1 \mathrm{H}), 4.67(\mathrm{~s}, 2 \mathrm{H}), 4.23 \sim 4.16(\mathrm{~m}, 3 \mathrm{H}), 3.58$ (t, $J=6.8 \mathrm{~Hz}$, $1 \mathrm{H}), 3.44$ (t, $J=6.8 \mathrm{~Hz}, 2 \mathrm{H}), 3.27$ (dd, $J=14.0,3.7 \mathrm{~Hz}$, $1 \mathrm{H}), 2.91 \sim 2.82(\mathrm{~m}, 1 \mathrm{H}), 1.67 \sim 1.26(\mathrm{~m}, 4 \mathrm{H}), 1.24(\mathrm{t}, J=$ $6.5 \mathrm{~Hz}, 3 \mathrm{H})$. HR-MS calcd for $\mathrm{C}_{32} \mathrm{H}_{33} \mathrm{~F}_{3} \mathrm{~N}_{3} \mathrm{O}_{7}[\mathrm{M}+\mathrm{H}]^{+}$ 628.2265 , found 628.2249 .

2.2.5 5-对羟基芐基- $N^{3}$-(5-乙氧羰基-4-芐氧氨基)戊基2,4-味唑二酮苯甲酸酯的甲酰化反应

$100 \mathrm{~mL}$ 单口瓶中加入 $0.68 \mathrm{~g}(1.2 \mathrm{mmol})$ BHIA3 及 $10 \mathrm{~mL}$ 二氯甲烷, 搅拌溶解后, 加入 $4 \mathrm{~mL}$ 无水甲酸, 摚 匀后再滴加 $1 \mathrm{~mL}$ 乙酸䣶, $5 \mathrm{~min}$ 内滴完, 常温摚拌 $2 \mathrm{~h}$, TLC 监测原料荧光点消失, 体系置于冷水浴中, 加入 10 $\mathrm{mL}$ 水, 用 $20 \%$ 氢氧化钠溶液调 $\mathrm{pH}$ 至 6 7, 二氯甲烷萃 三次，有机相经无水硫酸钠干燥，减压脱除溶剂得 0.66 $\mathrm{g}$ 粗品, 残余物经硅胶柱层析 (100 200 目), 以二氯甲 烷/无水甲醇/乙酸 (体积比为 $150: 2: 1$ ) 为洗脱剂洗脱, 得微无色粘稠液体 BHIA9 $0.60 \mathrm{~g}$, 收率为 $84 \%$. 采用相 同方法合成化合物 BHIA7 BHIA12.

5-对羟基苄基- $N^{3}$-(5-乙氧羰基-4- $N$-甲酰基-芐氧氨 基)戊基-2,4-咪唑二酮苯甲酸酯(BHIA7): 无色油状液 体, 收率 73\%. ${ }^{1} \mathrm{H}$ NMR $\left(300 \mathrm{MHz}, \mathrm{CDCl}_{3}\right) \delta: 8.24(\mathrm{~s}$, $1 \mathrm{H}), 8.19(\mathrm{~d}, J=7.3 \mathrm{~Hz}, 1 \mathrm{H}), 7.67 \sim 7.62(\mathrm{~m}, 1 \mathrm{H}), 7.54 \sim$ $7.49(\mathrm{~m}, 2 \mathrm{H}), 7.38 \sim 7.35(\mathrm{~m}, 5 \mathrm{H}), 7.25(\mathrm{~d}, J=8.5 \mathrm{~Hz}$, $2 \mathrm{H}), 7.17$ (d, $J=8.5 \mathrm{~Hz}, 2 \mathrm{H}), 5.40$ (s, 1H), 4.99 (s, 2H), 4.84 (s, 1H), $4.25 \sim 4.17$ (m, 3H), 3.49 (brs, $2 \mathrm{H}), 3.32$ (dd, $J=13.6,3.4 \mathrm{~Hz}, 1 \mathrm{H}), 2.84(\mathrm{dd}, J=13.6,8.8 \mathrm{~Hz}, 1 \mathrm{H}), 1.97$ (brs, 2H), 1.60 (brs, 2H), 1.28 (t, $J=7.1 \mathrm{~Hz}, 3 \mathrm{H})$. HR-MS calcd for $\mathrm{C}_{32} \mathrm{H}_{34} \mathrm{~N}_{3} \mathrm{O}_{8}[\mathrm{M}+\mathrm{H}]^{+}$588.2340, found 588.2339 .

5-对羟基苄基- $N^{3}$-(5-乙氧羰基-4- $N$-甲酰基-芐氧氨 基)戊基-2,4-咪唑二酮-2-甲氧基苯甲酸酯(BHIA8): 无 色油状液体, 收率 87\%. ${ }^{1} \mathrm{H}$ NMR $\left(300 \mathrm{MHz}, \mathrm{CDCl}_{3}\right) \delta$ : $8.23(\mathrm{~s}, 1 \mathrm{H}), 8.00(\mathrm{~d}, J=6.9 \mathrm{~Hz}, 1 \mathrm{H}), 7.56 \sim 7.51(\mathrm{~m}, 1 \mathrm{H})$, 7.35 (brs, $5 \mathrm{H}$ ), $7.26 \sim 7.01(\mathrm{~m}, 6 \mathrm{H}), 5.49(\mathrm{~s}, 1 \mathrm{H}), 4.98$ (s, $2 \mathrm{H}), 4.84(\mathrm{~s}, 1 \mathrm{H}), 4.25 \sim 4.17(\mathrm{~m}, 3 \mathrm{H}), 3.91(\mathrm{~s}, 3 \mathrm{H}), 3.49$ 
(brs, 2H), 3.32 (dd, $J=13.6,3.4 \mathrm{~Hz}, 1 \mathrm{H}), 2.84$ (dd, $J=$ 13.6, $8.8 \mathrm{~Hz}, 1 \mathrm{H}), 1.96$ (brs, 2H), 1.61 (brs, 2H), 1.28 (t, $J=7.1 \mathrm{~Hz}, 3 \mathrm{H})$. HR-MS calcd for $\mathrm{C}_{33} \mathrm{H}_{36} \mathrm{~N}_{3} \mathrm{O}_{9}[\mathrm{M}+\mathrm{H}]^{+}$ 618.2446, found 618.2434.

5 -对着基苄基- $N^{3}$-(5-乙氧羰基-4- $N$-甲酰基-芐氧氨 基)戊基-2,4-咪唑二酮-2-氟苯甲酸酯(BHIA9): 无色油 状液体，收率 $84 \% .{ }^{1} \mathrm{H}$ NMR $\left(300 \mathrm{MHz}^{-} \mathrm{CDCl}_{3}\right) \delta: 8.24$ $(\mathrm{s}, 1 \mathrm{H}), 8.06(\mathrm{dd}, J=7.8,2.0 \mathrm{~Hz}, 1 \mathrm{H}), 7.64 \sim 7.56(\mathrm{~m}, 1 \mathrm{H})$, 7.37 (brs, $5 \mathrm{H}), 7.29 \sim 7.16(\mathrm{~m}, 6 \mathrm{H}), 5.87(\mathrm{~s}, 1 \mathrm{H}), 4.98(\mathrm{~s}$, $2 \mathrm{H}), 4.83(\mathrm{~s}, 1 \mathrm{H}), 4.24 \sim 4.17(\mathrm{~m}, 3 \mathrm{H}), 3.47$ (brs, $2 \mathrm{H}), 3.24$ $(\mathrm{dd}, J=13.6,3.4 \mathrm{~Hz}, 1 \mathrm{H}), 2.87(\mathrm{dd}, J=13.6,8.8 \mathrm{~Hz}, 1 \mathrm{H})$, 1.95 (brs, 2H), 1.62 (brs, 2H), 1.26 (t, $J=7.1 \mathrm{~Hz}$, $3 \mathrm{H})$. HR-MS calcd for $\mathrm{C}_{32} \mathrm{H}_{33} \mathrm{FN}_{3} \mathrm{O}_{8}[\mathrm{M}+\mathrm{H}]^{+}$606.2246, found 606.2236 .

5-对着基茮基- $N^{3}$-(5-乙氧羰基-4- $N$-甲酰基-芐氧氨 基)戊基-2,4-咪坐二酮-2-氯苯甲酸酯(BHIA10): 无色油 状液体, 收率 77\%. ${ }^{1} \mathrm{H}$ NMR $\left(300 \mathrm{MHz}, \mathrm{CDCl}_{3}\right) \delta: 8.23$ $(\mathrm{s}, 1 \mathrm{H}), 8.03(\mathrm{~d}, J=7.8 \mathrm{~Hz}, 1 \mathrm{H}), 7.55 \sim 7.46(\mathrm{~m}, 2 \mathrm{H}), 7.39$ (brs, 5H), $7.26 \sim 7.17(\mathrm{~m}, 5 \mathrm{H}), 5.87(\mathrm{~s}, 1 \mathrm{H}), 4.98(\mathrm{~s}, 2 \mathrm{H})$, $4.84(\mathrm{~s}, 1 \mathrm{H}), 4.30 \sim 4.16(\mathrm{~m}, 3 \mathrm{H}), 3.47$ (brs, $2 \mathrm{H}), 3.22(\mathrm{dd}$, $J=13.6,3.4 \mathrm{~Hz}, 1 \mathrm{H}), 2.85$ (dd, $J=13.6,8.8 \mathrm{~Hz}, 1 \mathrm{H}), 1.94$ (brs, 2H), 1.61 (brs, 2H), 1.24 (t, $J=7.1 \mathrm{~Hz}, 3 \mathrm{H}$ ). HR-MS calcd for $\mathrm{C}_{32} \mathrm{H}_{33} \mathrm{ClN}_{3} \mathrm{O}_{8}[\mathrm{M}+\mathrm{H}]^{+}$622.1951, found 622.1941 .

5-对羟基苄基- $N^{3}$-(5-乙氧羰基-4- $N$-甲酰基-芐氧氨 基)戊基-2,4-咪唑二酮-2-甲基苯甲酸酯(BHIA11): 无色 油状液体, 收率 71\%. ${ }^{1} \mathrm{H} \mathrm{NMR}\left(300 \mathrm{MHz}, \mathrm{CDCl}_{3}\right) \delta: 8.23$ $(\mathrm{s}, 1 \mathrm{H}), 8.14(\mathrm{~d}, J=7.5 \mathrm{~Hz}, 1 \mathrm{H}), 7.50 \sim 7.45(\mathrm{~m}, 1 \mathrm{H}), 7.36$ (brs, 5H), $7.30 \sim 7.14(\mathrm{~m}, 6 \mathrm{H}), 5.73(\mathrm{~s}, 1 \mathrm{H}), 4.98(\mathrm{~s}, 2 \mathrm{H})$, $4.87(\mathrm{~s}, 1 \mathrm{H}), 4.24 \sim 4.12(\mathrm{~m}, 3 \mathrm{H}), 3.49$ (brs, $2 \mathrm{H}), 3.24$ (dd, $J=13.6,3.4 \mathrm{~Hz}, 1 \mathrm{H}), 2.83$ (dd, $J=13.6,8.8 \mathrm{~Hz}, 1 \mathrm{H}), 2.65$ (s, 3H), 1.97 (brs, 2H), 1.65 (brs, 2H), 1.27 (t, $J=7.2 \mathrm{~Hz}$, $3 \mathrm{H})$. HR-MS calcd for $\mathrm{C}_{33} \mathrm{H}_{36} \mathrm{~N}_{3} \mathrm{O}_{8}[\mathrm{M}+\mathrm{H}]^{+}$602.2497, found 602.2482 .

5 -对着基芳基- $N^{3}$-(5-乙氧羰基-4- $N$-甲酰基-芐氧氨 基)戊基-2,4-咪唑二酮-2-三氟甲基苯甲酸酯(BHIA12): 无色油状液体, 收率 $82 \% .{ }^{1} \mathrm{H} \mathrm{NMR}\left(300 \mathrm{MHz}, \mathrm{CDCl}_{3}\right) \delta$ : $8.25(\mathrm{~s}, 1 \mathrm{H}), 7.97 \sim 7.94(\mathrm{~m}, 1 \mathrm{H}), 7.83 \sim 7.80(\mathrm{~m}, 1 \mathrm{H})$, $7.71 \sim 7.66(\mathrm{~m}, 1 \mathrm{H}), 7.36$ (brs, 5H), $7.26 \sim 7.16(\mathrm{~m}, 4 \mathrm{H})$, $5.97(\mathrm{~s}, 1 \mathrm{H}), 4.97(\mathrm{~s}, 2 \mathrm{H}), 4.86(\mathrm{~s}, 1 \mathrm{H}), 4.24 \sim 4.12(\mathrm{~m}$, 3H), 3.49 (brs, 2H), 3.25 (dd, $J=13.6,3.4 \mathrm{~Hz}, 1 \mathrm{H}), 2.87$ (dd, $J=13.6,8.5 \mathrm{~Hz}, 1 \mathrm{H}$ ), 1.98 (brs, 2H), 1.62 (brs, 2H), 1.26 (t, $J=7.2 \mathrm{~Hz}, 3 \mathrm{H}$ ). HR-MS calcd for $\mathrm{C}_{33} \mathrm{H}_{33} \mathrm{~F}_{3} \mathrm{~N}_{3} \mathrm{O}_{8}$ $[\mathrm{M}+\mathrm{H}]^{+}$656.2214, found 656.2202 .
2.2.65-对羟基芐基- $\mathrm{N}^{3}$-(5-乙氧羰基-4-芐氧氨基)戊基2,4-咪唑二酮苯甲酸酯的脱芐基反应

在 $100 \mathrm{~mL}$ Schlenk 瓶中, 将 $0.60 \mathrm{~g}(1.0 \mathrm{mmol})$ BHIA9 溶于 $20 \mathrm{~mL}$ 无水甲醇中，加入 $10 \% \mathrm{Pd} / \mathrm{C} 120 \mathrm{mg}$, 将瓶中空气反复置换为氢气后, 开始缓慢通入氢气, 10 $\mathrm{h}$ 后, TLC 监测原料点消失, 停止搅拌, 过滤, 减压脱除 溶剂, 残余物经柱层析分离纯化, 以二氯甲烷/无水甲 醇/乙酸(体积比为 $150: 2: 1$ ) 为洗脱剂洗脱得无色油状 液体 BHIA15, $0.26 \mathrm{~g}$, 收率 51\%。采用相同方法合成化 合物 BHIA13 BHIA18.

5 -对羟基苠基- $N^{3}$-(5-乙氧羰基-4- $N$-甲酰基羟氨基) 戊基-2,4-咪唑二酮苯甲酸酯(BHIA13): 无色油状液体, 收率 60\%. ${ }^{1} \mathrm{H}$ NMR $\left(300 \mathrm{MHz}, \mathrm{CDCl}_{3}\right) \delta: 8.48$ (brs, 0.5H), $8.18 \sim 8.14(\mathrm{~m}, 2 \mathrm{H}), 8.00$ (brs, $0.5 \mathrm{H}), 7.66 \sim 7.61(\mathrm{~m}, 1 \mathrm{H})$, $7.54 \sim 7.47(\mathrm{~m}, 2 \mathrm{H}), 7.28 \sim 7.10(\mathrm{~m}, 4 \mathrm{H}), 6.55$ (brs, $1 \mathrm{H})$, 4.30 (brs, $1 \mathrm{H}), 4.20 \sim 4.14$ (m, 2H), 3.48 (brs, $2 \mathrm{H}), 3.28 \sim$ $3.20(\mathrm{~m}, 1 \mathrm{H}), 3.02 \sim 2.92(\mathrm{~m}, 1 \mathrm{H}), 1.91$ (brs, 2H), 1.59 (brs, $2 \mathrm{H}), \quad 1.31 \sim 1.21(\mathrm{~m}, 3 \mathrm{H})$. HR-MS calcd for $\mathrm{C}_{25} \mathrm{H}_{28} \mathrm{~N}_{3} \mathrm{O}_{8}[\mathrm{M}+\mathrm{H}]^{+}$498.1871, found 498.1863.

5 -对羟基苠基- $N^{3}$-(5-乙氧羰基-4- $N$-甲酰基羟氨基) 戊基-2,4-咪唑二酮-2-甲氧基苯甲酸酯(BHIA14): 无色 油状液体, 收率 $38 \% .{ }^{1} \mathrm{H}$ NMR $\left(300 \mathrm{MHz}, \mathrm{CDCl}_{3}\right) \delta: 8.45$ (brs, $0.5 \mathrm{H}), 8.08 \sim 8.04$ (m, 1H), 8.01 (brs, 0.5H), 7.57 $7.53(\mathrm{~m}, 1 \mathrm{H}), 7.27 \sim 7.24(\mathrm{~m}, 2 \mathrm{H}), 7.12 \sim 7.00(\mathrm{~m}, 4 \mathrm{H})$, 6.79 (brs, 1H), 4.35 (brs, 1H), $4.21 \sim 4.16$ (m, 2H), 3.91 (s, $3 \mathrm{H}), 3.48$ (brs, $2 \mathrm{H}), 3.28 \sim 3.20(\mathrm{~m}, 1 \mathrm{H}), 3.01 \sim 2.92(\mathrm{~m}$, 1H), 1.87 (brs, 2H), 1.51 (brs, $2 \mathrm{H}), 1.25 \sim 1.18$ (m, 3H). HR-MS calcd for $\mathrm{C}_{26} \mathrm{H}_{30} \mathrm{~N}_{3} \mathrm{O}_{9}[\mathrm{M}+\mathrm{H}]^{+}$528.1977, found 528.1967.

5-对着基苠基- $N^{3}$-(5-乙氧羰基-4- $N$-甲酰基羟氨基) 戊基-2,4-咪唑二酮-2-氟苯甲酸酯(BHIA15): 无色油状 液体，收率 51\%. ${ }^{1} \mathrm{H}$ NMR $\left(300 \mathrm{MHz}, \mathrm{CDCl}_{3}\right) \delta: 8.47$ (brs, $0.5 \mathrm{H}), 8.17 \sim 8.09(\mathrm{~m}, 1 \mathrm{H}), 8.02$ (brs, $0.5 \mathrm{H}), 7.54 \sim 7.50$ $(\mathrm{m}, 1 \mathrm{H}), 7.30 \sim 7.17(\mathrm{~m}, 6 \mathrm{H}), 6.25$ (brs, 1H), 4.29 (brs, $1 \mathrm{H}), 4.21 \sim 4.16(\mathrm{~m}, 2 \mathrm{H}), 3.46$ (brs, $2 \mathrm{H}), 3.26 \sim 3.20(\mathrm{~m}$, $1 \mathrm{H}), 3.00 \sim 2.90(\mathrm{~m}, 1 \mathrm{H}), 1.86$ (brs, 2H), 1.48 (brs, 2H), $1.29 \sim 1.20(\mathrm{~m}, 3 \mathrm{H})$. HR-MS calcd for $\mathrm{C}_{25} \mathrm{H}_{27} \mathrm{FN}_{3} \mathrm{O}_{8}[\mathrm{M}+$ $\mathrm{H}]^{+}$516.1777, found 516.1762.

5 -对着基苠基- $N^{3}$-(5-乙氧羰基-4- $N$-甲酰基羟氨基) 戊基-2,4-咪唑二酮-2-氯苯甲酸酯(BHIA16): 无色油状 液体, 收率 44\%. ${ }^{1} \mathrm{H}$ NMR $\left(300 \mathrm{MHz}, \mathrm{CDCl}_{3}\right) \delta: 8.46$ (brs, $0.5 \mathrm{H}), 8.18 \sim 8.15(\mathrm{~m}, 1 \mathrm{H}), 8.02$ (brs, $0.5 \mathrm{H}), 7.64 \sim 7.61$ $(\mathrm{m}, 1 \mathrm{H}), 7.53 \sim 7.38(\mathrm{~m}, 2 \mathrm{H}), 7.27 \sim 7.24(\mathrm{~m}, 2 \mathrm{H}), 7.19 \sim$ 7.13 (m, 2H), 6.51 (brs, 1H), 4.27 (brs, 1H), 4.26 4.12 $(\mathrm{m}, 2 \mathrm{H}), 3.47$ (brs, $2 \mathrm{H}), 3.28 \sim 3.22(\mathrm{~m}, 1 \mathrm{H}), 2.97 \sim 2.90$ 
(m, 1H), 1.85 (brs, 2H), 1.52 (brs, 2H), 1.33 1.22 (m, $3 \mathrm{H})$. HR-MS calcd for $\mathrm{C}_{25} \mathrm{H}_{27} \mathrm{ClN}_{3} \mathrm{O}_{8}[\mathrm{M}+\mathrm{H}]^{+} 532.1475$, found 532.1481 .

\section{5-对着基芳基- $\mathrm{N}^{3}$-(5-乙氧羰基-4- $\mathrm{N}$-甲酰基羟氨基)} 戊基-2,4-咪唑二酤-2-甲基苯甲酸酯(BHIA17)：无色油 状液体，收率 41\%. ${ }^{1} \mathrm{H}$ NMR $\left(300 \mathrm{MHz}, \mathrm{CDCl}_{3}\right) \delta: 8.47$ (brs, $0.5 \mathrm{H}), 8.15 \sim 8.11(\mathrm{~m}, 1 \mathrm{H}), 7.98$ (brs, 0.5H), $7.51 \sim$ $7.45(\mathrm{~m}, 1 \mathrm{H}), 7.34 \sim 7.25(\mathrm{~m}, 4 \mathrm{H}), 7.15 \sim 7.10(\mathrm{~m}, 2 \mathrm{H})$, 6.50 (brs, 1H), 4.29 (brs, 1H), $4.22 \sim 4.14$ (m, 2H), 3.48 (brs, $2 \mathrm{H}), 3.28 \sim 3.20(\mathrm{~m}, 1 \mathrm{H}), 2.99 \sim 2.93(\mathrm{~m}, 1 \mathrm{H}), 2.65$ (s, 3H), 1.90 (brs, 2H), 1.54 (brs, 2H), $1.28 \sim 1.21$ (m, 3H). HR-MS calcd for $\mathrm{C}_{26} \mathrm{H}_{30} \mathrm{~N}_{3} \mathrm{O}_{8}[\mathrm{M}+\mathrm{H}]^{+}$512.2027, found 512.2018 .

\section{5 -对着基苠基- $\mathrm{N}^{3}$-(5-乙氧羰基-4- $\mathrm{N}$-甲酰基羟氨基)} 戊基-2,4-咪唑二酮-2-三氟甲基苯甲酸酯(BHIA18): 无 色油状液体，收率 58\%. ${ }^{1} \mathrm{H}$ NMR $\left(300 \mathrm{MHz} \mathrm{CDCl}_{3}\right) \delta$ : 8.46 (brs, 0.5H), 8.05 8.01 (m, 1H), 7.95 (brs, 0.5H), $7.84 \sim 7.81(\mathrm{~m}, 1 \mathrm{H}), 7.70 \sim 7.67(\mathrm{~m}, 1 \mathrm{H}), 7.39 \sim 7.27(\mathrm{~m}$, $2 \mathrm{H}), 7.19 \sim 7.13(\mathrm{~m}, 2 \mathrm{H}), 6.51$ (brs, $1 \mathrm{H}), 4.29$ (brs, 1H), $4.25 \sim 4.14(\mathrm{~m}, 2 \mathrm{H}), 3.46$ (brs, $2 \mathrm{H}), 3.29 \sim 3.20(\mathrm{~m}, 1 \mathrm{H})$, $3.00 \sim 2.91(\mathrm{~m}, 1 \mathrm{H}), 1.89$ (brs, $2 \mathrm{H}), 1.58$ (brs, 2H), $1.31 \sim$ $1.21(\mathrm{~m}, 3 \mathrm{H})$. HR-MS calcd for $\mathrm{C}_{26} \mathrm{H}_{27} \mathrm{~F}_{3} \mathrm{~N}_{3} \mathrm{O}_{8}[\mathrm{M}+\mathrm{H}]^{+}$ 566.1745 , found 566.1733 .

\section{辅助材料(Supporting Information) 化合物的 ${ }^{1} \mathrm{H}$ NMR} 和 HR-MS 谱图. 这些材料可以免费从本刊网站 (http://sioc-journal.cn/)上下载.

\section{References}

[1] (a) Fonné-Pfister, R.; Chemla, P.; Ward, E.; Girardet, M.; Kreuz, K. E.; Honzatko, R. B.; Fromm, H. J.; Schar, H. P.; Grütter, M. G.; Cowan-Jacob, S. W. Proc. Natl. Acad. Sci. U. S. A. 1996, 93, 9431. (b) Poland, B. W.; Silva, M. M.; Serra, M. A.; Cho, Y.; Kim, K. H.; Harris, E. M.; Honzatko, R. B. J. Biol. Chem. 1993, 268, 25334. (c) Poland, B. W.; Hou, Z. L.; Bruns, C.; Fromm, H. J.; Honzatko, R. B. J. Biol. Chem. 1996, 271, 15407.

(d) Cseke, C. T.; Gerwick, B. C.; Crouse, B. C.; Murdoch, M. G.; Green, S. B.; Heim, D. R. Pestic. Biochem. Physiol. 1996, 55, 210. (e) Prade, L.; Cowan-Jacob, S. W.; Chemla, P.; Potter, S.; Ward, E.; Fonne-Pfister, R. J. Mol. Biol. 2000, 296, 569.

(f) Poland, B. W.; Lee, S.; Subramanian, M. V.; Siehl, D. L.; Anderson, R. J.; Fromm, H. J.; Honzatko, R. B. Biochemistry 1996, 35,15753 .

[2] Tibrewal, N.; Elliott, G. I. Bioorg. Med. Chem. Lett. 2011, 21, 517.

[3] Boitz, J. M.; Strasser, R.; Yates, P. A.; Jardim, A.; Ullman, B. J. Biol. Chem. 2013, 288, 8977.

[4] Wiest, A.; McCarthy, A. J.; Schnittker, R.; McCluskey, K. J. Genet. 2012, 91, 199

[5] Wrasidlo, W.; Elliott, G. I.; Carson, D. A.; Rosenbach, M. WO 2009023495, 2009 [Chem. Abstr. 2009, 150, 252697].

[6] (a) Gray, R. A.; Gauger, G. W.; Dulaney, E. L.; Kaczka, E. A.;
Woodruff, H. B. Plant Physiol. 1964, 39, 204. (b) Hatch, M. D. Phytochemistry 1967, 6, 115.

[7] Nakajima, N.; Itoi, K.; Takamatsu, Y.; Kinoshita, T.; Okazaki, T.; Kawakubo, K.; Shindo, M.; Honma, T.; Tohjigamori, M.; Haneishi, T. J. Antibiot. 1991, 44, 293.

[8] (a) Wang, J. M.; Han, J. T.; Kuang, Y.; Jin, S. H.; Wang, M. A. Chin. J. Pestic. Sci. 2008, 10, 392 (in Chinese).

(王进敏，韩金涛，匡宇，金淑惠，王明安，农药学学报，2008， 10, 392.)

(b) Wang, J. M.; Kuang, Y.; Han, J. T.; Jin, S. H.; Wang, M. A. Chin. J. Pestic. Sci. 2009, 11, 1 (in Chinese).

(王进敏，匡宇，韩金涛，金淑惠，王明安，农药学学报，2009，11, 1.)

[9] (a) Walters, E. W.; Lee, S-F.; Niderman, T.; Bernasconi, P.; Subramanian, M. V.; Siehl, D. L. Plant Physiol. 1997, 114, 549.

(b) Siehl, D. L.; Subramanian, M. V.; Walters, E. W.; Lee, S. F.; Anderson, F. J.; Toschi, A. G. Plant Physiol. 1996, 110, 753.

[10] Wang, Y. P.; Wang, M. A.; Du, F. P.; Li, X. D.; Yao, G. W.; Zhang, P. D.; Lu, H. Z. Chem. J. Chin. Univ. 2010, 31, 336 (in Chinese).

(汪月鹏, 王明安, 杜风沛, 李向东, 姚广伟, 张潘丹, 路慧哲, 高等学校化学学报, 2010, 31, 336.)

[11] Lienhard, G. E.; Secemski, I. I. J. Biol. Chem. 1973, 248, 1121.

[12] (a) Xu, Z. H.; Wang, J. M.; Han, J. T.; Liu, B.; Wang, M. A. Chin. J. Org. Chem. 2012, 32, 2134 (in Chinese).

(徐志红, 王进敏, 韩金涛, 刘斌, 王明安, 有机化学, 2012, 32, 2134.)

(b) Han, J. T.; Wang, J. M.; Dong, H. B.; Xu, Z. H.; Liu, B.; Wang, M. A. Chin. J. Org. Chem. 2013, 33, 596 (in Chinese).

(韩金涛, 王进敏, 董宏波, 徐志红, 刘斌, 王明安, 有机化学, 2013, 33, 596.)

(c) Wang, J. M.; Han, J. T.; Zhang, C. Y.; Qiu, L. H.; Wang, M. A. Chin. J. Org. Chem. 2010, 30, 72 (in Chinese).

(王进敏，韩金涛，张春艳，邱立红，王明安，有机化学，2010, 30,72 .)

(d) Han, J. T.; Wang, J. M.; Chen, S. C.; Qiu, L. H.; Wang, M. A. Chin. J. Org. Chem. 2010, 30, 691 (in Chinese)

(韩金涛，王进敏，陈守聪，邱立红，王明安，有机化学， 2010, 30, 691.)

(e) Xu, Z. H.; Liu, B.; Dong, H. B.; Wang, M. A. Chin. J. Org. Chem. 2014, 34, 2517 (in Chinese).

(徐志红, 刘斌, 董宏波, 王明安. 有机化学, 2014, 34, 2517.)

(f) Xu, Z. H.; Dong, H. B.; Liu, B.; Kong, L. Q.; Wang, M. A. Chin. J. Org. Chem. 2015, 35, 411 (in Chinese).

(徐志红, 董宏波, 刘斌, 孔令青, 王明安, 有机化学, 2015, 35, 411.)

(g) Han, J. T.; Dong, H. B.; Xu, Z. H.; Wang, J. M.; Wang, M. A. Int. J. Mol. Sci. 2013, 14, 19526.

(h) Han, J. T.; Dong, H. B.; Xu, Z. H.; Lei, J. P.; Wang, M. A. Int. J. Mol. Sci. 2013, 14, 12484.

(i) Han, J. T.; Dong, H. B.; Wang, J. M.; Lei, J. P.; Wang, M. A.; Fang, J. X. Molecules 2011, 16, 2833.

[13] (a) Medzihradszky, D.; Chen, S. L.; Kenyon, G. L.; Gibson, B. W. J. Am. Chem. Soc. 1994, 116, 9413.

(b) Darja, L.; Marje, L.; Indrek, V.; Norbert, K.; Gerda, R.; Jevgenia, R.; Erki, E.; Uri, A.; Dirk, B. J. Med. Chem. 2009, 52, 308.

(c) Enkvist, E.; Lavogina, D.; Raidaru, G.; Vaasa, A.; Viil, I.; Lust, M.; Viht, K.; Uri, A. J. Med. Chem. 2006, 49, 7150.

(d) Van Wandelen, L. T. M.; Poot, A. J.; Ismail-Ali, A. F.; van Ufford, H. C. Q.; Vijftigschild, L. A. W.; Beekman, J. M.; Martin, N. I.; Ruijtenbeek, R.; Liskamp, R. M. J. Chem. Biol. 2013, 8, 1479.

(e) Van Ameijde, J.; Poot, A. J.; Van Wandelen, L. T. M.; Wammes, A. E. M.; Ruijtenbeek, R.; Rijkers, D. T. S.; Liskamp, R. M. J. Org. 
Biomol. Chem. 2010, 8, 1629.

[14] Hanessian, S.; Lu, P. P.; Sancéau, J. Y.; Chemla, P.; Gohda, K.; Fonne-Pfister, R.; Prade, L.; Cowan-Jacob, S. W. Angew. Chem., Int. Ed. 1999, 38, 3160.

[15] Zhang, Y.; Elliott, G. I.; Saldanha, A.; Carson, D. A.; Wrasidlo, W. Beilstein J. Org. Chem. 2010, 6, 742.

[16] Wolfe, S.; Akuche, C.; Ro, S.; Wilson, M.; Kim, C.; Shi, Z. Can. J. Chem. 2003, 81, 915.

[17] Gentili, D.; Macchia, M.; Menchini, E.; Nencetti, S.; Orlandini, E.;
Rossello, A.; Broccali, G.; Limonta, D. Farmaco 1999, 54, 224.

[18] Xue, S.; Lu, S. X.; Liu, X. M.; Zhao, Q. Chin. J. Magn. Reson. 1999, 16, 131 (in Chinese)

(薛松, 陆世维, 刘秀梅, 赵琦, 波谱学杂志, 1999, 16, 131.)

[19] (a) Ksander, G. M.; McMurry, J. E.; Johnson, M. A. J. Org. Chem. 1977, 42, 1180.

(b) Lawson, P. J.; McCarthy, M. G.; Sargeson, A. M. J. Am. Chem. Soc. 1982, 104, 6710.

(Li, L.; Lu, Z.) 